\title{
IDENTIDAD, LENGUA Y MARCO LEGAL EN CATALUÑA
}

Francesc Mercadé

\section{IDENTIDAD Y LENGUA}

\section{Reflexiones previas}

En el contexto de las identidades colectivas en España, la diversidad lingǘstica tiene un peso fundamental. La lengua es - por ejemplo- uno de los atributos diferenciadores de las denominadas nacionalidades históricas. Pero ¿cuál es su papel en el sentimiento de pertenencia? ¿Qué sentido tiene esta variable en las definiciones de integración cultural o en la socialización política?

Cualquier respuesta a los interrogantes anteriores debe circunscribirse a una situación geográfica y a un tiempo histórico determinado. Por ejemplo, me parece un error metodológico - sesgado ideológicamente- haber introducido la lengua como variable principal para estudiar el proceso de integración de los inmigrantes que llegaron a Cataluña bajo el franquismo. Evidentemente, el reto de la "integración" se situaba entonces básicamente en transformaciones profundas que cambiaban el ámbito de referencia (de rural a urbano), los espacios y ritmos de trabajo (del agrario al industrial), las relaciones humanas y las pautas de convivencia (de familia extensa a familia nuclear, del grupo amplio de referencia a la disgregación, del pueblo a la ciudad). 
Vivir en algún suburbio de la gran ciudad, trasladarse en complicados y lentos sistemas de transporte, tener puestos de trabajo que implican tareas repetitivas y que se desarrollan en espacios cerrados, sentir profundos cambios en las referencias cultarales más generales (secularización, politización, individualismo) y modificar totalmente los usos y las costumbres de la vida cotidiana, eran los cambios fundamentales que vivían las personas que habían marchado de los pueblos de Andalucía o Murcia (por ejemplo) para instalarse en un barrio marginal de Barcelona.

El contacto de los inmigrantes con la cultura autóctona era muy pequeño, y el papel de la lengua catalana en "la integración» era prácticamente nulo. El catalán no aparecía más que en momentos tangenciales y accesorios de la vida cotidiana de un catalán de adopción. Tanto el tiempo de trabajo como los momentos de ocio se desarrollan en espacios abiertos en los que nunca era necesaria la comprensión -y menos aún el uso- de la lengua propia de Cataluña. En las fábricas, en los bares, en el cine, en la televisión se practicaba un estricto monolingüismo en castellano.

Durante las últimas etapas del régimen franquista y, sobre todo, después de la restauración de la democracia, el uso del catalán ha ido ganando espacios, no sólo en cuanto a la extensión de su uso, sino fundamentalmente en lo que respecta a su definitiva normalización. El reconocimiento constitucional de la pluralidad lingüística de España va más allá de una simple constatación, para convertir a las diversas lenguas en patrimonio colectivo.

En este sentido, en la Cataluña actual los usos del catalán se hallan mucho más extendidos y han penetrado en todos los ámbitos de la convivencia cotidiana. La enseñanza en las escuelas de la lengua propia de Cataluña, la existencia de dos canales de televisión en catalán y la penetración de esta lengua en otros, así como el uso cada vez más amplio por parte de los demás medios de comunicación, han sentado las bases para una nueva situación lingüística en Cataluña.

\section{Los datos oficiales}

El tema de la lengua, en Cataluña, es pues hoy un factor de gran interés para conocer la realidad y para descubrir aspectos relacionados con las identidades colectivas en nuestro país. La normalización lingüística tiene su máxima expresión en la utilización del catalán en todas las diferentes situaciones de la vida de cada día.

El último censo lingüístico elaborado por el Consorci d'Informació i Documentació a partir de la información de los Padrones Municipales de 1986 ha puesto de manifiesto los niveles de conocimiento del catalán ${ }^{1}$ en

1 Las indicaciones que constan en el Padrón Municipal para contestar estas cuestiones del conocimiento del catalán especifican lo siguiente: entender el catalán significa ser capaz 
el ámbito de la Comunidad Autónoma de Cataluña (cuadro 1). Más de un 90 por 100 de la población residente en Cataluña entiende el catalán, aunque únicamente el 64 por 100 lo sabe hablar, el 60 por 100 lo sabe leer y el 31 por 100 es capaz de escribirlo.

Desagregando por provincias esta información, podemos constatar una distribución desigual de los datos del censo linguístico. Barcelona en el ámbito en el que el catalán es menos conocido: un 89 por 100 lo entienden, un 60 por 100 lo saben hablar, un 58 por 100 lo saben leer y un 30 por 100 lo saben escribir. Lérida es la provincia en la que el conocimiento de la lengua propia de Cataluña es más elevado: un 96 por 100 "entienden", un 83 por 100 "hablan", un 72 por 100 "leen" y un 37 por 100 "escriben"; bastante parecidos son los datos de implantación del catalán en Gerona, que son ligeramente inferiores (en uno o dos puntos) con la excepción del conocimiento del catalán escrito que es superior en dos puntos. Tarragona se encuentra en un punto intermedio entre Barcelona $y$ las dos provincias que acabamos de comentar.

\section{CUADRO 1}

\section{Niveles de conocimiento del catalán* \\ Niveles de conocimiento del caralan*}

Lo entiende

Lo sabe hablat

Lo sabe leer

Lo sabe escribir

No consta
* Tanto por ciento de la población global, mayor de dos años.

FUENTE: Padrones Municipales de Habitantes de 1986.

CATA-

\begin{tabular}{ccccc} 
Barcelona & Gerona & Lérida & Tarragona & $\begin{array}{c}\text { CATA } \\
\text { LUN } A\end{array}$ \\
\hline 89,0 & 95,1 & 96,3 & 92,8 & 90,3 \\
59,8 & 80,1 & 82,8 & 72,9 & 64,0 \\
58,2 & 70,7 & 71,6 & 63,7 & 60,5 \\
30,1 & 39,3 & 37,1 & 32,0 & 31,5 \\
0,3 & 0,4 & 0,8 & 0,4 & 0,3 \\
& & & & \\
\hline
\end{tabular}

De todas maneras, ya comentábamos anteriormente que la normalización lingüística es un proceso abierto y que, por lo tanto, debe ser analizado desde una perspectiva dinámica. En el cuadro 2 podemos observar la evolución de la compresión del catalán entre los censos de 1981 y 1986. El crecimiento más espectacular (de 12 puntos, del 72 por 100 al 89 por 100 ), se ha producido lógicamente en Barcelona, que era la provincia más castellanizada y en la que queda todavía mucho terreno por recorrer.

de comprender una conversación sobre un tema corriente en esta lengua; saber leerlo quiere decir comprender textos como anuncios o noticias de periódico; saber hablarlo consiste en poder mantener una charla en catalán sobre cuestiones no especializadas; escribirlo significa saber redactar notas o escribir postales en catalán relativamente correcto. 


\section{CUADRO 2}

Evolución de la comprensión del catalán 1981-1986*

\begin{tabular}{|c|c|c|c|c|c|}
\hline & Barcelona & Gerona & Lérida & Tarragona & $\begin{array}{l}C A T A- \\
L U N A\end{array}$ \\
\hline ………………… & 89,0 & 95,1 & 96,3 & 92,8 & 90,3 \\
\hline Comprensión 1981 .............................. & 77,1 & 90,7 & 91,8 & 86,0 & 79,8 \\
\hline Diferencia $1986-1981 \ldots \ldots \ldots \ldots \ldots \ldots \ldots$ & 11,9 & 4,4 & 4,5 & 6,9 & 10,5 \\
\hline
\end{tabular}

* Tanto por ciento de la población global, mayor de dos años.

Fuente: Padrones Municipales de Habitantes de 1986.

El siguiente ámbito en el que se han consolidado grandes avances es Tarragona (del 86 por 100 al 93 por 100 de comprensión del catalán). A continuación quedan Gerona y Lérida con pequeños pero significativos avances superiores al 4 por 100 .

\section{Los datos propios}

Ya hemos visto cómo de los datos oficiales se desprende que un 64 por 100 de los catalanes saben hablar en catalán; de todas maneras, según los datos de la encuesta, tan sólo un 55 por 100 utilizan la lengua propia de Cataluña en sus conversaciones cotidianas.

La información de los Padrones Municipales respecto a los usos lingüísticos de las distintas provincias catalanas también contrasta con nuestra información "de primera mano" que confirma y matiza algunos aspectos. En el cuadro 3 podemos constatar cómo el castellano es la lengua

\section{CUADRO 3}

\section{Lengua utilizada con más frecuencia}

\begin{tabular}{|c|c|c|c|c|c|}
\hline & Barcelona & Gerona & Lérida & Tarragona & $\begin{array}{l}C A T A- \\
L U \tilde{N} A\end{array}$ \\
\hline (1) & 45,9 & 71,9 & 76,7 & 67,0 & 55,5 \\
\hline 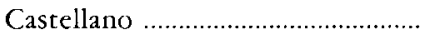 & 51,7 & 26,1 & 21,9 & 30,2 & 42,5 \\
\hline 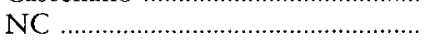 & 2,3 & 2,0 & 1,4 & 2,8 & 2,0 \\
\hline 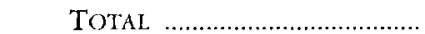 & 100 & 100 & 100 & 100 & 100 \\
\hline
\end{tabular}

Fuente: Encuesta de GABISE, S. A., a la población de Cataluña, febrero 1988. 
utilizada con más frecuencia en Barcelona (52 por 100). Al contrario, la provincia más "catalanizada" es Lérida, donde casi el 77 por 100 de sus habitantes utilizan habitualmente el catalán. En Gerona ( 72 por 100) y en Tarragona (67 por 100) también domina el catalán como primera lengua de comunicación.

Es importante remarcar las diferencias que existen entre los datos oficiales - que se refieren a conocimiento del catalán-y la información primaria que presentamos, que concierne a los usos reales de la lengua en las situaciones de la vida diaria. El tamaño de municipio es una variable independiente relacionada con el grado de conocimiento del catalán. Cuanto más pequeño es el núcleo de residencia, mayor es el uso del catalán entre sus habitantes. En concreto - cuadro 4- en los municipios menores de 2.000 habitantes, más del 96 por 100 de la población utiliza el catalán en sus relaciones sociales.

\section{CUADRO 4}

Lengua de uso más frecuente, según tamaño de municipio

\begin{tabular}{|c|c|c|c|c|c|c|}
\hline & \multicolumn{5}{|c|}{ NUMERO DE HABITANTES } & \multirow[b]{2}{*}{$\begin{array}{l}C A T A \\
L U \bar{N} A\end{array}$} \\
\hline & $\begin{array}{r}\text { Hasta } \\
2.000\end{array}$ & $\begin{array}{l}\text { De } 2.001 \\
\text { a } 10.000\end{array}$ & $\begin{array}{c}\text { De } 10.001 \\
\text { a } 50.000\end{array}$ & $\begin{array}{l}\text { De } 50.001 \\
\text { a } 100.000\end{array}$ & $\begin{array}{l}\text { Más de } \\
10.000\end{array}$ & \\
\hline 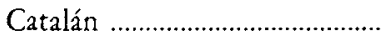 & 96,2 & 72,3 & 52,5 & 39,5 & 45,8 & 55,6 \\
\hline Castellano & 3,2 & 25,4 & 47,0 & 58,9 & 51,6 & 42,5 \\
\hline NC & 0,6 & 2,3 & 0,5 & 1,6 & 2,6 & 2,0 \\
\hline 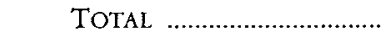 & 100 & 100 & 100 & 100 & 100 & 100 \\
\hline
\end{tabular}

Fuente: Encuesta de GABISE, S. A., a la población de Cataluña, febrero 1988.

Los municipios más castellanizados son los comprendidos entre 50.001 y 100.000 habitantes; esto puede explicarse por los procesos de desviación de las migraciones masivas de las grandes ciudades hacia núcleos intermedios, que tienen como resultado el hecho de desbordar su capacidad de integración lingüística y cultural. En las poblaciones mayores de 100.000 habitantes se recupera parcialmente el hábito de hablar en catalán.

$\mathrm{Si}$ nos referimos a la variable edad - cuadro 5- podemos constatar cómo las edades más altas (mayores de 56 años) tienen un nivel superior de utilización del catalán. Las edades intermedias (de 36 a 55 años) utilizan en menor proporción la lengua propia de Cataluña, seguramente porque es la franja que más ha experimentado la influencia de los procesos migrato- 


\section{CUADRO 5}

Lengua utilizada con más frecuencia, según la edad de los entrevistados

\begin{tabular}{|c|c|c|c|c|c|c|c|}
\hline & \multicolumn{6}{|c|}{$E D A D$} & \multirow[b]{2}{*}{ CATALUÑ $A$} \\
\hline & De 18 a 25 & De 26 a 35 & De 36 a 45 & De 46 a 55 & De $56 a 65$ & Más de 65 & \\
\hline 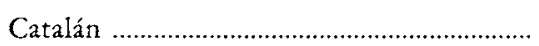 & 56,3 & 53,2 & 43,7 & 49,6 & 61,6 & 68,5 & 55,6 \\
\hline 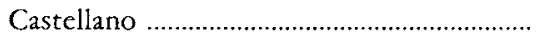 & 41,0 & 42,9 & 54,0 & 49,6 & 37,4 & 30,1 & 42,4 \\
\hline NC & 2,4 & 3,9 & 2,3 & 0,8 & 0,9 & 1,4 & 2,0 \\
\hline TOTAL …1. & 100 & 100 & 100 & 100 & 100 & 100 & \\
\hline
\end{tabular}

Fuente: Encuesta de GABISE, S. A. a la población de Cataluña, febrero 1988. 
rios. Finalmente, las generaciones más jóvenes recuperan el uso de la lengua catalana de manera clara, influenciados por la enseñanza del catalán en la escuela y por los variados caminos de la normalización. Entre todo el conjunto de agentes que consolidan esta tendencia positiva, destaca el papel de los medios de comunicación y especialmente la televisión en catalán.

En el cuadro 6 podemos observar la lógica, relación entre el sentimiento de identidad y los usos lingüísticos de los catalanes. De los entrevistados que hablan habitualmente en catalán, muy. pocos (2,5 por 100) se definen como españoles a secas, un tanto por ciento parecido $(2,4$ por 100$)$ se consideran más españoles que catalanes.

Igualmente, es interesante constatar cómo casi un 30 por 100 de los catalano-parlantes se identifican a sí mismos como tan españoles como catalanes. Por último, un 2,5 por 100 de los que hablan catalán se sienten más catalanes que españoles y un 41 por 100 únicamente catalanes. Queda claro que existe una relación directa entre el sentimiento subjetivo de identidad y el grado de utilización del catalán.

Por último, me gustaría destacar la relación existente entre el uso de la lengua y la posición política. En el cuadro 7 podemos comprobar las distintas prácticas lingüísticas de los entrevistados diseccionadas según el voto decidido en febrero de 1988 respecto a las Elecciones Autonómicas de mayo del mismo año. Las preferencias políticas tienen una distinta expresión entre los castellanoparlantes y los catalanoparlantes.

Por una parte el electorado de CiU utiliza fundamentalmente el catalán en su vida cotidiana, y la mayor parte de castellanoparlantes que tenían su voto decidido optaban por el PSC (PSC-PSOE). El caso más extremo es el de Esquerra Republicana de Catalunya (ERC): este partido tiene la práctica totalidad de sus electores situados en la franja de los entrevistados que utilizan el catalán como lenga más frecuente.

Concretamente, de cada 100 ciudadanos que hablan normalmente el catalán, 11 votan al PSC, 34 a CiU, 1 a AP, 0,6 al CDS, 3 a Iniciativa per Catalunya (IC), 2 a ERC. Se observa, pues, una delimitación entre la posición lingüística del voto de izquierdas (PSC y IC ) y el comportamiento en los usos de la lengua de los electores que optan por la derecha nacionalista (CiU). El caso de ERC, AP y el CS sigue una trayectoria distinta. 


\section{CUADRO 6}

Lengua de uso más frecuente, según sentimiento de identidad

\begin{tabular}{|c|c|c|c|c|c|c|c|}
\hline & \multicolumn{6}{|c|}{ SENTIMIENTO DE IDENTIDAD } & \multirow[b]{2}{*}{ TOTAL } \\
\hline & Español & $\begin{array}{l}\text { Más español } \\
\text { que catalán }\end{array}$ & $\begin{array}{l}\text { Tan español } \\
\text { como catalán }\end{array}$ & $\begin{array}{l}\text { Más catalán } \\
\text { que español }\end{array}$ & Catalán & $\begin{array}{l}\text { Ni catalán } \\
\text { ni español }\end{array}$ & \\
\hline Catalán & 2,5 & 2,4 & 28,4 & 24,9 & 41 & 0,7 & 100 \\
\hline 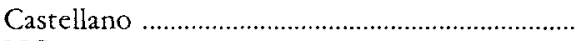 & 32,9 & 13,5 & 42,1 & 5,4 & 3,7 & 2,5 & 100 \\
\hline NC & 27,6 & 13,8 & 31,0 & 6,9 & 6,9 & 13,8 & 100 \\
\hline 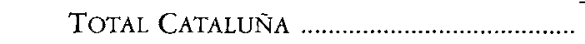 & 15,9 & 7,3 & 34,3 & 16,3 & 24,5 & 1,8 & 100 \\
\hline
\end{tabular}

FUente: Encuesta de GABISE, S. A. a la población de Cataluña, febrero 1988.

\section{CUADRO 7}

Lengua utilizada con más frecuencia, según voto decidido

\begin{tabular}{|c|c|c|c|c|c|c|c|c|c|c|}
\hline & $\begin{array}{c}P S C \\
(P S C-P S O E)\end{array}$ & $\mathrm{CiU}$ & $A P$ & $C D S$ & PSUC & $E R C$ & Otros & $\begin{array}{l}\text { Absten- } \\
\text { ción }\end{array}$ & $N S / N C$ & TOTAL \\
\hline Catalán & 10,6 & 34,2 & 1,1 & 0,6 & 3,3 & 1,9 & 1,1 & 5,8 & 41,3 & 100 \\
\hline Castellano & 28,3 & 7,9 & 3,8 & 0,3 & 4,9 & 0,0 & 0,6 & 6,2 & 47,9 & 100 \\
\hline NC & 10,3 & 13,8 & 0,0 & 0,0 & 6,9 & 0,0 & 3,4 & 3,4 & 62,1 & 100 \\
\hline Total Cataluñ & 18,1 & 22,6 & 2,2 & 0,5 & 4,1 & 1,1 & 0,9 & 5,9 & 44,5 & 100 \\
\hline
\end{tabular}

FUENTE: Encuesta de GABISE, S. A., a la población de Cataluña, febrero 1988. 


\section{IDENTIDAD Y LENGUA.}

\section{ANEXO: OTROS DATOS DE LA ENCUESTA}

\section{CUADRO 1.I}

Lengua de uso más frecuente en relación a la opinión respecto a la evolución de Cataluña en los últimos años

\begin{tabular}{|c|c|c|c|c|}
\hline \multirow[b]{2}{*}{ Situación en Cataluña } & \multicolumn{3}{|c|}{ LENGUA MAS FRECUENTE } & \multirow[b]{2}{*}{ TOTAL } \\
\hline & Catalán & Castellano & $N C$ & \\
\hline Ha mejorado & 54,2 & 42,5 & 51,7 & 49,2 \\
\hline Sigue igual .. & 36,6 & 42,5 & 31,0 & 39,0 \\
\hline 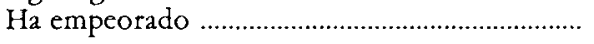 & 9,2 & 14,9 & 17,2 & 11,8 \\
\hline . & 100 & 100 & 100 & 100 \\
\hline
\end{tabular}

Fuente: Encuesta de GABISE, S. A., a la población de Cataluña, febrero 1988.

\section{CUADRO 2.I}

Lengua de uso más frecuente en relación al grado de satisfacción respecto a la gestión de la Generalitat

\begin{tabular}{|c|c|c|c|c|}
\hline \multirow[b]{2}{*}{ Nivel de satisfacción } & \multicolumn{3}{|c|}{ LENGUA MAS FRECUENTE } & \multirow[b]{2}{*}{ TOTAL } \\
\hline & Catalán & Castellano & $N C$ & \\
\hline Muy satisfecho .......... & 5,6 & 2,9 & 0,0 & 4,3 \\
\hline 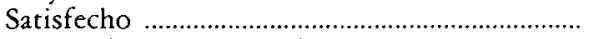 & 46,2 & 28,5 & 17,2 & 38,2 \\
\hline 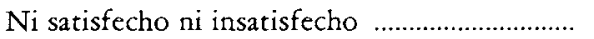 & 36,7 & 49,4 & 55,2 & 42,4 \\
\hline Insatisfecho & 9,4 & 16,0 & 17,2 & 12,4 \\
\hline 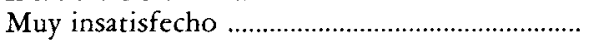 & 2,1 & 3,2 & 10,3 & 2,7 \\
\hline .1. & 100 & 100 & 100 & 100 \\
\hline
\end{tabular}

Fuente: Encuesta de GABISE, S. A., a la población de Cataluna, febrero 1988. 


\section{CUADRO 3.I}

Lengua de uso más frecuente, según opinión respecto a las relaciones Generalitat-Gobierno Central

\begin{tabular}{|c|c|c|c|c|}
\hline \multirow[b]{2}{*}{ Relaciones Generalitat-Madrid } & \multicolumn{3}{|c|}{ LENGUA MAS FRECUENTE } & \multirow[b]{2}{*}{ TOTAL } \\
\hline & Catalán & Castellano & $N C$ & \\
\hline Pésimas & 8,6 & 4,4 & 6,9 & 6,8 \\
\hline Malas & 32,5 & 29,8 & 20,7 & 27,3 \\
\hline 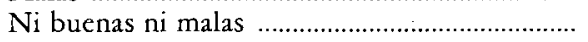 & 40,2 & 50,5 & 37,9 & 44,5 \\
\hline 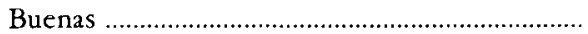 & 7,9 & 11,9 & 31,0 & 10,0 \\
\hline Optimas & 1,8 & 2,4 & 0,0 & 2,0 \\
\hline $\mathrm{NC}$ & 9,0 & 10,0 & 3,4 & 9,3 \\
\hline TOTAL & 100 & 100 & 100 & 100 \\
\hline
\end{tabular}

Fuente: Encuesta de GABISE, S. A., a la población de Cataluña, febrero 1988.

\section{CUADRO 4.I}

Lengua de uso más frecuente, según su opinión respecto al Estatuto de Cataluña

\begin{tabular}{|c|c|c|c|c|}
\hline \multirow[b]{2}{*}{ Opinión Estatuto } & \multicolumn{3}{|c|}{ LENGUA MAS FRECUENTE } & \multirow[b]{2}{*}{ TOTAL } \\
\hline & Catalán & Castellano & $N C$ & \\
\hline 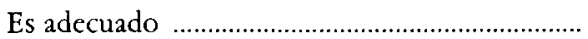 & 29,6 & 47,9 & 32,1 & 37,4 \\
\hline Se debería ampliar & 68,3 & 46,1 & 60,7 & 58,7 \\
\hline Se debería recortar & 2,1 & 6,0 & 7,1 & 3,8 \\
\hline TOTAL & 100 & 100 & 100 & 100 \\
\hline
\end{tabular}

FueNTE: Encuesta de GABISE, S. A., a la población de Cataluña, febrero 1988.

\section{CUADRO 5.I}

Lengua de uso más frecuente en relación al partido más próximo a la manera de pensar del entrevistado (simpatía)

\begin{tabular}{|c|c|c|c|c|}
\hline \multirow[b]{2}{*}{ Partido más próximo } & \multicolumn{3}{|c|}{ LENGUA MAS FRECUENTE } & \multirow[b]{2}{*}{ TOTAL } \\
\hline & Catalán & Castellano & NC & \\
\hline PSC & 15,2 & 45,6 & 32,1 & 28,4 \\
\hline 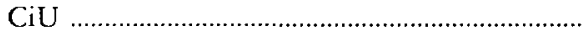 & 45,0 & 10,6 & 25,0 & 30,0 \\
\hline (2) & 2,3 & 5,2 & 3,6 & 3,6 \\
\hline 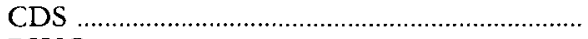 & 1,5 & 2,1 & 0,0 & 1,7 \\
\hline 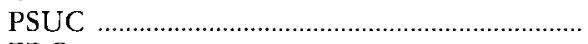 & 3,9 & 6,3 & 14,3 & 5,2 \\
\hline ERC & 4,7 & 0,3 & 0,0 & 2,7 \\
\hline Otros & 3,2 & 2,3 & 7,1 & 2,9 \\
\hline NC & 24,2 & 27,6 & 17,9 & 25,5 \\
\hline . & 100 & 100 & 100 & 100 \\
\hline
\end{tabular}

Fuente: Encuesta de GABISE, S. A., a la población de Cataluña, febrero 1988. 


\section{LA IDENTIDAD Y EL MARCO LEGAL}

\section{El marco legal y la vida cotidiana}

Ya he señalado la importancia del desarrollo del Estado de las Autonomías como nuevo marco de convivencia en España. Las identidades colectivas van transformándose lenta pero inexorablemente, a partir de una nueva estructura administrativa y de un nuevo entramado legal. A pesar de que - como veremos- muchas veces el debate parlamentario no ha sabido llegar a la opinión pública, la verdad es que los cambios introducidos han impactado en la vida cotidiana de los ciudadanos.

Han pasado todavía pocos años y, sin embargo, las identidades colectivas en España están viviendo una profunda transformación. Después del largo período franquista parécía imposible mover la anquilosada y centralista maquinaria del Estado en el sentido de reconocer la diversidad de los pueblos que componen el mosaico español. Mucho más complicado parecía reconocer que la pluralidad es precisamente riqueza y patrimonio colectivo, que la defensa de todas y cada una de las culturas es una obligación común.

A pesar de los complejos pactos de la transición política, de las inercias y de la ambigüedad de nuestro texto constitucional - en algunos puntos de más difícil consenso-, es innegable que la consolidación del Estado de las Autonomías ha significado una transformación de nuestro marco de referencia, así como de nuestra vida cotidiana.

El "nuevo mapa de España" ha reconocido algunas realidades nacionales existentes (nacionalidades según la Constitución), pero ha topado con algunas naciones más dinámicas (históricas), otras más pausadas, e incluso unas que se sitúan "en otra dimensión" respecto al modelo propuesto.

Muchas veces, las "batallas" políticas coyunturales (electorales) hacen olvidar las perspectivas a largo plazo. La construcción de un marco de identidades compartidas y solidarias debe de constituir el objetivo prioritario de España en el final de la década de los años ochenta. Será muy difícil que en el futuro volvamos a tener una oportunidad histórica de re-diseñar los signos de identidad y de afianzar una nueva solidaridad colectiva.

Por otro lado, situados en el contexto de la Comunidad Autónoma Catalana, la aprobación del Estatuto y el desarrollo posterior de sucesivas leyes - La Ley de Normalización Lingüística y la Ley de Ordenación del Territorio, por ejemplo-, han contribuido a modificar opiniones, pero sobre todo actitudes. En las páginas que siguen vamos a comentar los interesantes resultados de sucesivos sondeos de opinión que referían algunas de sus preguntas al Estatuto de Cataluna, a la nueva Ley de Ordenación del Territorio, $y$, en concreto, a la propuesta de creación de una provincia zinica en el actual territorio de la Comunidad Autónoma Catalana. 


\section{Los catalanes y la reforma del Estatuto de Autonomía}

Más de la mitad de la población de Cataluña es favorable a ampliar las competencias del actual gobierno catalán y, en consecuencia, varía con buenos ojos la reforma del Estatuto. Esta información - recogida en el cuadro 8- me parece sumamente interesante, puesto que recoge de alguna manera el clima político existente en Cataluña y que - tal como hemos visto- se produce en otras Comunidades Autónomas.

Me parece claro que la misma implantación del Estado Autonómico ha generado un conjunto de expectativas tanto en las nacionalidades históricas como en las de reciente creación. En los datos podemos comprobar cómo un amplio margen de entrevistados (58,6 por 100) considera que se ha de ampliar el marco del autogobierno catalán, frente a un 37,5 por 100 que consideran que la situación actual es adecuada.

En el otro extremo, las respuestas que se inclinan por una restricción de las competencias en un futuro, no tienen una significación especial, puesto que ni tan sólo alcanzan el 4 por 100. De todas maneras, vale la pena remarcar que en la provincia de Barcelona los partidarios de disminuir las competencias se acerca al 5 por 100.

Precisamente, las diferencias provinciales en cuanto a la opinión sobre el Estatuto son relativamente importantes: Lérida (64 por 100) y Tarragona ( 72 por 100) son las zonas geográficas más críticas, puesto que acumulan una gran mayoría de opiniones a favor de ampliar el actual Estatuto de Autonomía.

\section{CUADRO 8}

\section{Opiniones sobre el actual Estatuto de Autonomía de Cataluña}

\begin{tabular}{|c|c|c|c|c|c|}
\hline & Barcelona & Gerona & Lérida & Tarragona & $\begin{array}{l}C A T A- \\
L U \tilde{N} A\end{array}$ \\
\hline (2) & 37,1 & 46,4 & 34,9 & 25,5 & 37,5 \\
\hline 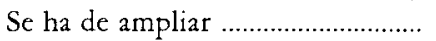 & 57,9 & 50,5 & 63,7 & 71,8 & 58,6 \\
\hline 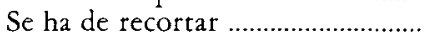 & 4,8 & 3,1 & 1,5 & 2,7 & 3,9 \\
\hline
\end{tabular}

Fuente: Encuesta, S. A., a la población de Cataluña, febrero 1988. 
El futuro de las Diputaciones $y$ de las comarcas

En una reciente encuesta (marzo de 1988) realizada por la empresa Información Cartográfica y de Base, S. A. (ICB, S. A.), con la colaboración de EMOPUBLICA en el trabajo de campo $^{2}$, queda claro nuevamente cómo los catalanes desconocen la realidad de las diputaciones. Menos de un 36 por 100 de los entrevistados conocen el número exacto de estas entidades públicas existente en Cataluna (cuadro 9); la mayor parte de las respuestas declaran no saber, o no contestan, la citada pregunta.

Por otro lado, de los mismos datos se desprende que casi un 40 por 100 de la población de Catalunya está en contra de la desaparición de las diputaciones. Si analizamos las cifras a nivel provincial (cuadro 10) podemos comprobar cómo Tarragona y Lérida son las zonas más reacias a la eliminación de las diputaciones (43 y 41 por 100, respectivamente).

Tal como veremos en el siguiente apartado, la nueva organización comarcal va a traer consigo fuertes cambios en la estructura de las instituciones y del poder político en Cataluña. Concretamente en el cuadro 11 podemos observar cómo tan sólo un 4 por 100 de los catalanes conocen con precisión el número de comarcas que configuran la realidad actual de Cataluña; un amplísimo 78 por 100 "no sabe» o "no contesta» la cantidad de comarcas que configuran el mapa catalán, y tan sólo un 4 por 100 conoce con precisión el número exacto (38 comarcas en la distribución de 1936).

\section{CUADRO 9}

Número de diputaciones que existen en Cataluña

\begin{tabular}{|c|c|c|c|c|c|}
\hline & Barcelona & Gerona & Lérida & Tarragona & $\begin{array}{l}C A T A- \\
L U \tilde{N} A\end{array}$ \\
\hline Menos de cuatro' ${ }^{1}$ & 6,8 & 3,2 & 1,2 & 1,5 & 5,6 \\
\hline Cuatro & 33,7 & 38,3 & 43,8 & 45,3 & 35,7 \\
\hline Más de cuatro ${ }^{2}$ & 1,3 & 1,0 & 0,5 & 0,2 & 1,1 \\
\hline 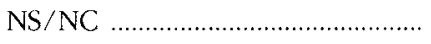 & 58,2 & 57,5 & 54,5 & 53,0 & 57,6 \\
\hline 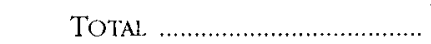 & 100 & 100 & 100 & 100 & 100 \\
\hline
\end{tabular}

1 Agrupa las respuestas incorrectas de una a tres.

2 Agrupa las respuestas incorrectas de cinco a "más de ocho".

FueNTE: Encuesta ICB, S. A., febrero-marzo 1988.

2 Ficha técnica: 2.800 entrevistas realizadas en 138 municipios en Cataluña. Error muestral de 1,89 por 100 para un nivel de confianza del 95 por 100 . Rutas aleatorias. Trabajo de campo realizado entre el 22 de febrero y el día 3 de marzo de 1988. 


\section{CUADRO 10}

Opiniones sobre la desaparición de las diputaciones

\begin{tabular}{|c|c|c|c|c|c|}
\hline & Barcelona & Gerona & Lérida & Tarragona & $\begin{array}{l}C A T A- \\
L U \tilde{N} A\end{array}$ \\
\hline Deberían desaparecer ........................ & 20,7 & 16,7 & 16,7 & 16,0 & 19,7 \\
\hline No deberían desaparecer .............. & 38,6 & 38,3 & 40,7 & 43,0 & 39,1 \\
\hline NS/NC & 40,7 & 45,0 & 42,7 & 41,0 & 41,2 \\
\hline TOTAL & 100 & 100 & 100 & 100 & 100 \\
\hline
\end{tabular}

Fuente: Encuesta ICB, S. A., febrero-marzo 1988.

CUADRO 11

Niveles de comarcas que existen en Cataluña

\begin{tabular}{|c|c|c|c|c|c|}
\hline & Barcelona & Gerona & Lérida & Tarragona & $\begin{array}{l}C A T A- \\
L U \tilde{N} A\end{array}$ \\
\hline Correcto & 4,0 & 4,5 & 3,8 & 5,5 & 4,2 \\
\hline Incorrecto & 20,1 & 18,3 & 13,0 & 16,6 & 19,1 \\
\hline NS/NC & 75,9 & 77,2 & 83,2 & 77,9 & 76,7 \\
\hline 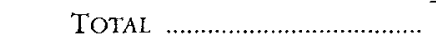 & 100 & 100 & 100 & 100 & 100 \\
\hline
\end{tabular}

Fuente: Encuesta ICB, S. A., febrero-marzo 1988.

La mitad de los catalanes desconocen la LOT

Con fuerte controversia parlamentaria se aprobó en Cataluña la Ley de Ordenación del Territorio (LOT). Con este nuevo marco legal desaparecía la Corporación Metropolitana de Barcelona y se recreaba un abanico de comarcas que reconstruían el mapa catalán bajo la República. De todas maneras, la nueva propuesta de organización implica también la modificación del peso de las distintas fuerzas políticas; la comarcalización de Cataluña da más peso a los territorios del interior (en los que domina la derecha), frente a los núcleos urbanos más poblados (tradicionalmente de voto más «izquierdista»).

La sorpresa de los datos que comentamos reside en conocer que esta polémica política, prácticamente no traspasó las paredes del Parlament. Más de la mitad de los catalanes no conocen la Ley de Ordenación del Territorio (LOT), y tan sólo la rechazan un 6,5 por 100 . 
En el conjunto de Cataluña, el 48 por 100 de los hombres y el 64 por 100 de las mujeres desconocen la realiad de la LOT y, por lo tanto, no pueden pronunciarse sobre esta Ley que ha nacido entre polémicas de partidos. De toda manera, un 23 por 100 de los ciudadanos manifiestan que están de acuerdo con la LOT, frente a un 6,5 por 100 que se muestran contratios (cuadro 12).

\section{CUADRO 12}

Opiniones respecto a la Ley de Ordenación del Territorio (LOT)

\begin{tabular}{|c|c|c|c|c|c|}
\hline & Barcelona & Gerona & Lérida & Tarragona & $\begin{array}{l}\text { CATA- } \\
L U \tilde{N} A\end{array}$ \\
\hline 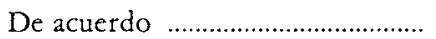 & 20,4 & 33,7 & 35,7 & 27,6 & 23,4 \\
\hline 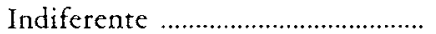 & 11,2 & 24,4 & 14,4 & 13,7 & 12,8 \\
\hline En desacuerdo & 6,6 & 5,2 & 7,8 & 5,4 & 6,5 \\
\hline No la conoce & 61,7 & 36,7 & 42,1 & 53,3 & 57,3 \\
\hline
\end{tabular}

Fuente: Encuesta de GABISE, S. A., a la población de Cataluña, marzo 1988.

En general, tenemos muy poco conocimiento real sobre el impacto de la LOT en la vida y en las opiniones de los ciudadanos. La información que manejamos nos permite contrastar cómo los enfrentamientos entre partidos políticos no simpre tienen un reflejo paralelo en la opinión pública.

Parece muy claro - en los datos que comentamos - que cuando se pide a los ciudadanos de Cataluña su opinión respecto a la LOT, éstos no han percibido el impacto que esta Ley puede tener en la inmediata vida del "día a día" de los catalanes. En este sentido, cuando se desconocen algunos aspectos básicos de la obra del gobierno autónomo, es lógica la dificultad para recoger opiniones desfavorables sobre leyes concretas.

El problema fundamental sobre el que queremos llamar la atención es que un 62 por 100 de las respuestas en Barcelona, un 53 por 100 en Tarragona, un 42 por 100 en Lérida, y un 37 por 100 en Gerona no conocen absolutamente nada sobre esta cuestión. La edad es también una variable interesante a considerar en este tema: es curioso constatar cómo son precisamente los mayores de 56 años los más críticos respecto a la LOT, situándose claramente por encima de la media.

De todas maneras se ha de remarcar que las valoraciones más positivas se concentran en Lérida y en Gerona (un 36 por 100 y un 34 por 100 de opiniones favorables), frente a Tarragona (23 por 100), y sobre todo en Barcelona (20 por 100). La distribución tertitorial de las opiniones, según 
tamaño de municipio, no es tampoco homogénea. Los niveles más bajos de acuerdo con la LO'T se concentran en las ciudades de más de 100.000 habitantes, y las más altas cotas de acuerdo, en los pueblos más pequeños (de menos de 2.000) o en las ciudades intermedias (entre 50.000 y 100.000 habitantes).

Un tema más controvertido es la propuesta de organización de Cataluña en provincia única (cuadro 13). Ya comentábamos anteriormente la distancia que muchas veces existe entre el debate parlamentario y la opinión de los ciudadanos; la discusión sobre la unificación provincial de Barcelona ha sido, concretamente, el eje central de profundas discrepancias políticas $y$, en cambio, un 40 por 100 de los entrevistados se muestran "indiferentes" ante este proyecto.

El resto de los catalanes - que emiten su juicio sobre el tema- se presenta muy dividido en dos partes contrarias relativamente simétricas a favor y en contra. Un 35 por 100 de los entrevistados son favorables y un 25 por 100 se definen como contrarios. Es importante constatar cómo las zonas más industriales son menos favorables a la constitución de esta futura provincia única. Tarragona es la única zona geográfica en la que existen más opiniones desfavorables (36 por 100) que favorables (34 por 100); y Barcelona también concentra actitudes críticas, con el tanto por ciento más bajo de "favorables" (32 por 100), y también con un relativamente alto grado (28 por 100) de visiones negativas.

También queda claro en los resultados de la encuesta que comentamos que los habitantes de las grandes ciudades son los más críticos en cuanto a expresar su apoyo a la idea de provincia única. De manera parecida a la que antes comentábamos, son igualmente los hábitats más pequeños (de menos de 2.000 habitantes) y las ciudades en las que viven de 50.000 a 10.000 personas las que recogen las proporciones mayores de posiciones favorables.

\section{CUADRO 13}

Opiniones sobre la propuesta de organización de Cataluña como provincia única

\begin{tabular}{|c|c|c|c|c|c|}
\hline & Barcelona & Gerona & Lérida & Tarragona & $\begin{array}{l}\text { CATA- } \\
L U \tilde{N} A\end{array}$ \\
\hline 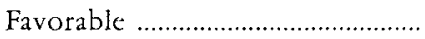 & 31,6 & 48,9 & 50,5 & 34,3 & 35,0 \\
\hline 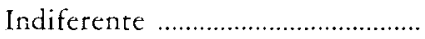 & 40,8 & 43,8 & 34,0 & 29,7 & 39,6 \\
\hline 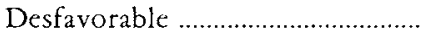 & 27,6 & 7,3 & 15,4 & 36,0 & 25,5 \\
\hline
\end{tabular}

FUENTE: Encuensta de GABISE, S. A., a la población de Cataluña, marzo 1988. 
En la Cataluña rural y con menos densidad de población, las opiniones son globalmente mucho más positivas para el proyecto de unificación provincial. Gerona y Lérida tienen a una mayoría, próxima al 50 por 100, que manifiesta una actitud favorable, frente a unos reducidos tantos por ciento que se muestran contrarios ( 15 por 100 en el caso de Lérida y 7 por 100 en el de Gerona).

Lo que parece mas sorprendente de los datos que comentamos es el análisis del impacto de los mensajes de los partidos y de las opciones ideológicas en las tan polémicas cuestiones de ordenación del territorio. En el cuadro 14 podemos ver cómo las posturas más favorables se sitúan - en primer lugar y a mucha distancia - entre los electores más próximos a ERC, un 42 por 100 de los cuales se manifiestan de acuerdo con la LOT. A continuación, con porcentajes próximos al 30 por 100 de visiones favorables se sitúan los simpatizantes de CiU y los de Iniciativa por Cataluña.

Los catalanes ideológicamente próximos a los socialistas, a Alianza Popular y al CDS se sitúan todos ellos en posiciones más críticas y reúnen, respectivamente, entre un 20 y un 25 por 100 de opiniones que expresan acuerdo con la Ley de Ordenación del Temitorio. Podemos comprobar, pues, que los partidos arrastran a una pequeña parte de sus simpatizantes hacia cuestiones ideológicas o de política general propuestas en sus idearios. Pero, tal como hemos visto - y todavía es más claro en el cuadro $15-$, la opinión de los ciudadanos es, en cierta medida, independiente a las presiones de los partidos.

\section{CUADRO 14}

La opinión respecto a la. LOT, de los simpatizantes de distintos partidos

\begin{tabular}{|c|c|c|c|c|c|}
\hline \multirow{2}{*}{$\begin{array}{c}\text { Proximidad o simpatia } \\
\text { ideológica }\end{array}$} & \multicolumn{4}{|c|}{ Opinión respecto a la $L O T$} & \multirow[b]{2}{*}{ TOTAL } \\
\hline & De acuerdo & Indiferente & En desacuerdo & No la conoce & \\
\hline ………….... & 21,7 & 12,0 & 5,4 & 60,9 & 100 \\
\hline $\mathrm{CiU}$ & 30,9 & 12,9 & 5,0 & 51,2 & 100 \\
\hline AP & 24,6 & 3,2 & 9,5 & 62,7 & 100 \\
\hline CDS & 23,6 & 17,0 & 5,5 & 53,9 & 100 \\
\hline IC & 28,0 & 11,0 & 11,6 & 49,4 & 100 \\
\hline (2) & 42,2 & 8,8 & 8,3 & 40,7 & 100 \\
\hline Otros & 32,0 & 13,2 & 16,2 & 38,6 & 100 \\
\hline $\mathrm{NC}$ & 12,4 & 14,4 & 6,4 & 66,8 & 100 \\
\hline TOTAL & 23,4 & 12,8 & 6,5 & 57,3 & 100 \\
\hline
\end{tabular}

FueNTE: Encuesta de GABISE, S. A., a la población de Cataluña, marzo 1988. 


\section{CUADRO 15}

Desglose según la simpatia ideologica de las diferentes posiciones respecto a la LOT

\begin{tabular}{|c|c|c|c|c|c|}
\hline \multirow{2}{*}{$\begin{array}{c}\text { Proximidad o simpatía } \\
\text { ideológica }\end{array}$} & \multicolumn{4}{|c|}{ Opinión respecto a la LOT } & \multirow[b]{2}{*}{ TOTAL } \\
\hline & De acuerdo & Indiferente & En desacuerdo & No la conoce & \\
\hline PSC & 21,6 & 22,5 & 20,4 & 25,4 & 23,8 \\
\hline ..................... & 42,5 & 33,3 & 25,7 & 29,4 & 32,8 \\
\hline AP & 3,0 & 0,8 & 4,4 & 3,2 & 3,0 \\
\hline CDS & 3,2 & 4,3 & 2,8 & 3,0 & 3,2 \\
\hline IC & 4,8 & 3,6 & 7,6 & 3,6 & 4,1 \\
\hline ERC & 7,4 & 2,9 & 5,5 & 3,0 & 4,2 \\
\hline Otros & 4,0 & 3,1 & 7,6 & 2,0 & 3,0 \\
\hline NC & 13,5 & 29,5 & 26,1 & 30,3 & 25,9 \\
\hline . & 100 & 100 & 100 & 100 & 100 \\
\hline
\end{tabular}

FUENTE: Encuesta de GABISE, S. A., a la población de Cataluña, marzo 1988.

Por último, en el cuadro 16 hemos contrastado las opiniones respecto al marco legal de la Ley de Ordenación del Territorio en relación a la valoración de la propuesta de constituir Cataluña en provincia única. Con esta información queda claro que no todos los que apoyan la LOT ven con buenos ojos la unificación provincial de Cataluña. En esta dirección se puede constatar cómo un amplio 21 por 100 de los que están de acuerdo con la Ley marco son desfavorables a la proposición de provincia única, y un 10 por 100 se muestran indiferentes. Desde la perspectiva contraria, los que no son partidarios de la LOT concentran igualmente un amplio 25 por 100 de lecturas favorables a la uniprovincialidad.

\section{CUADRO 16}

Opiniones respecto a la LOT en relación a la creación de provincia única

\begin{tabular}{|c|c|c|c|c|c|}
\hline \multirow{2}{*}{$\begin{array}{c}\text { Cataluña provincia } \\
\text { única }\end{array}$} & \multicolumn{4}{|c|}{ Opinión respecto a la $L O T$} & \multirow[b]{2}{*}{ TOTAL } \\
\hline & De acuerdo & Indiferente & En desacuerdo & No la conoce & \\
\hline 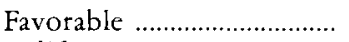 & 69,1 & 19,6 & 20,2 & 25,0 & 35,0 \\
\hline Indiferente .................................. & 9,8 & 62,6 & 11,2 & 50,8 & 39,4 \\
\hline Desfavorable ............................ & 21,1 & 17,8 & 68,6 & 24,2 & 25,6 \\
\hline TOTAL & 100 & 100 & 100 & 100 & 100 \\
\hline
\end{tabular}

Fuente: Encuesta de GABISE, S. A., a la población de Cataluña, marzo 1988. 
Estas reflexiones pueden servir para sensibilizar a los partidos respecto su influencia en la opinión pública. Los debates en el Parlamento, los nfrentamientos en los medios de comunicación, o las posturas radicalizalas en los sucesivos escenarios preelectorales, tendrían que servir para nformar al ciudadano de las alternativas reales de gobierno, y no de los slóganes electorales o de las pugnas internas.

\section{LA IDENTIDAD Y EL MARCO LEGAL.}

ANEXO: OTROS DATOS DE LA ENCUESTA

CUADRO 1.II

Opinión sobre la LOT, según el sexo de los entrevistados

\begin{tabular}{|c|c|c|c|}
\hline \multirow[b]{2}{*}{ Opinión sobre la $L O T$} & \multicolumn{2}{|c|}{$S E X O$} & \multirow[b]{2}{*}{ TOTAL } \\
\hline & Hombres & Mujeres & \\
\hline De acuerdo & 29,6 & 19,1 & 23,5 \\
\hline ndiferente .................. & 14,1 & 11,7 & 12,7 \\
\hline En desacuerdo & 8,0 & 5,4 & 6,5 \\
\hline No la conoce & 48,4 & 63,8 & 57,3 \\
\hline TOTAL & 100 & 100 & 100 \\
\hline
\end{tabular}

UENTE: Encuesta de GABISE, S. A., a la población de Cataluña, marzo 1988. 
CUADRO 2.11

Opinión sobre la LOT, según edad

\begin{tabular}{|c|c|c|c|c|c|c|c|}
\hline \multirow[b]{2}{*}{ Opinión sobre la $L O T$} & \multicolumn{6}{|c|}{$E D A D$} & \multirow[b]{2}{*}{ TOTAL } \\
\hline & De 18 a 25 & De 26 a 35 & De 36 a 45 & De 46 a 55 & De 55 a 65 & Más de 65 & \\
\hline De acuerdo & 25,1 & 25,1 & 29,0 & 25,8 & 18,0 & 18,5 & 23,5 \\
\hline 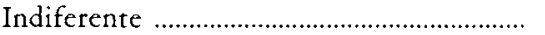 & 11,0 & 11,8 & 15,1 & 12,9 & 15,9 & 9,8 & 12,8 \\
\hline 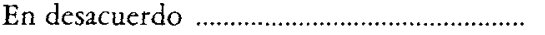 & 9,0 & 10,1 & 7,7 & 4,1 & 5,3 & 3,1 & 6,5 \\
\hline 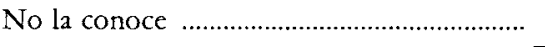 & 54,9 & 53,1 & 48,2 & 57,2 & 60,7 & 68,6 & 57,2 \\
\hline TOTAL & 100 & 100 & 100 & 100 & 100 & 100 & 100 \\
\hline
\end{tabular}

FUENTE: Encuesta de GABISE, S. A., a la población de Cataluña, marzo 1988.

\section{CUADRO 3.II}

Opinión sobre la LOT, según lugar de nacimiento

\begin{tabular}{|c|c|c|c|c|c|c|}
\hline \multirow[b]{2}{*}{ Opinión sobre la LOT } & \multicolumn{5}{|c|}{ LUGAR DE NACIMIENTO } & \multirow[b]{2}{*}{ TOTAL } \\
\hline & Municipio & Provincia & $\begin{array}{c}\text { Resto de } \\
\text { Cataluña }\end{array}$ & $\begin{array}{l}\text { Resto de } \\
\text { España }\end{array}$ & Extranjero & \\
\hline De acuerdo ....................... & 25,8 & 31,6 & 25,8 & 16,4 & 2,1 & 23,5 \\
\hline 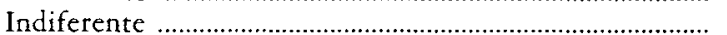 & 14,2 & 10,4 & 11,8 & 13,0 & 15,6 & 12,8 \\
\hline 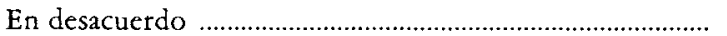 & 8,1 & 7,4 & 8,4 & 4,0 & 0,0 & 6,5 \\
\hline 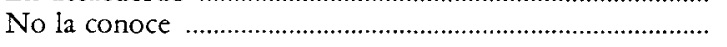 & 51,9 & 50,6 & 54,1 & 66,5 & 82,3 & 57,2 \\
\hline 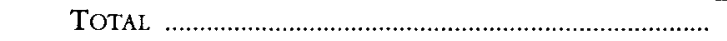 & 100 & 100 & 100 & 100 & 100 & 100 \\
\hline
\end{tabular}

Fuente: Encuesta de GABISE, S. A., a la población de Cataluña, marzo 1988. 


\section{CUADRO 4.II}

Opinión sobre la LOT, según tamaño del municipio

\begin{tabular}{|c|c|c|c|c|c|c|}
\hline \multirow[b]{2}{*}{ Valoración Autonomías } & \multicolumn{5}{|c|}{ NUMERO DE HABITANTES } & \multirow[b]{2}{*}{ TOTAL } \\
\hline & $\begin{array}{l}\text { Hasta } \\
2.000\end{array}$ & $\begin{array}{l}\text { De } 2.001 \\
\text { a } 10.000\end{array}$ & $\begin{array}{c}\text { De } 10.001 \\
\text { a } 50.000\end{array}$ & $\begin{array}{l}\text { De } 50.001 \\
\text { a } 100.000\end{array}$ & $\begin{array}{l}\text { Más de } \\
10.000\end{array}$ & \\
\hline 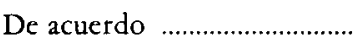 & 34,6 & 27,6 & 20,7 & 39,2 & 18,8 & 23,5 \\
\hline Indiferente. & 11,7 & 15,1 & 11,4 & 12,9 & 12,8 & 12,8 \\
\hline En desacuerdo & 6,7 & 6,9 & 5,2 & 4,6 & 7,1 & 6,5 \\
\hline No la conoce ......................... & 47,0 & 50,4 & 62,7 & 43,3 & 61,4 & 57,2 \\
\hline TOTAL & 100 & 100 & 100 & 100 & 100 & 100 \\
\hline
\end{tabular}

FuenTE: Encuesta de GABISE, S. A., a la población de Cataluña, marzo 1988.

\section{CUADRO 5.II}

Opinión sobre la LOT, según conocimiento de la lengua que deberán tener los inmigrantes

\begin{tabular}{|c|c|c|c|c|c|}
\hline \multirow[b]{2}{*}{ Opinión sobre la $L O T$} & \multicolumn{4}{|c|}{ CONOCIMIENTO DE LA LENGUA INMIGRANTES } & \multirow[b]{2}{*}{ TOTAL } \\
\hline & $\begin{array}{l}\text { Leery } \\
\text { escribir }\end{array}$ & Hablar & Entender & $\begin{array}{l}\text { Ni bablar, } \\
\text { ni entender }\end{array}$ & \\
\hline De acuerdo & 26,4 & 29,5 & 20,9 & 6,2 & 23,6 \\
\hline Indiferente & 12,3 & 16,2 & 11,2 & 9,7 & 12,8 \\
\hline En desacuerdo & 6,9 & 8,1 & 4,9 & 7,5 & 6,4 \\
\hline No la conoce & 54,3 & 46,2 & 63,0 & 76,6 & 57,3 \\
\hline TOTAL & 100 & 100 & 100 & 100 & 100 \\
\hline
\end{tabular}

Fuente: Encuesta de GABISE, S. A., a la población de Cataluña, marzo 1988. 


\section{CUADRO 6.II}

Opinión sobre la LOT, según preferencias en las publicaciones de la Generalitat

\begin{tabular}{|c|c|c|c|c|}
\hline \multirow[b]{2}{*}{ Opinión sobre la LOT } & \multicolumn{3}{|c|}{ Lengua preferida en las publicaciones } & \multirow[b]{2}{*}{ TOTAL } \\
\hline & Catalán & Castellano & Indiferente & \\
\hline De acuerdo & 29,3 & 19,7 & 14,7 & 23,5 \\
\hline 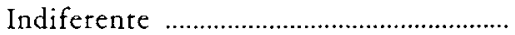 & 13,9 & 11,4 & 11,6 & 12,7 \\
\hline En desacuerdo & 7,4 & 4,9 & 6,6 & 6,5 \\
\hline 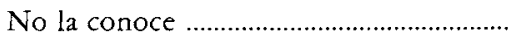 & 49,5 & 64,0 & 67,0 & 57,3 \\
\hline TOTAL & 100 & 100 & 100 & 100 \\
\hline
\end{tabular}

Fuente: Encuesta de GABISE, S. A., a la población de Cataluña, marzo 1988.

\section{CUADRO 7.II}

Opinión sobre la LOT, según el sentimiento predominante de identidad

\begin{tabular}{|c|c|c|c|c|c|c|c|}
\hline \multirow[b]{2}{*}{ Opinión sobre la LOT } & \multicolumn{6}{|c|}{ SENTIMIENTO DE IDENTIDAD } & \multirow[b]{2}{*}{ TOTAL } \\
\hline & Español & $\begin{array}{l}\text { Más español } \\
\text { que catalán }\end{array}$ & $\begin{array}{l}\text { Tan español } \\
\text { como catalán }\end{array}$ & $\begin{array}{l}\text { Más catalán } \\
\text { que español }\end{array}$ & Catalán & $\begin{array}{l}\text { Ni catalän, } \\
\text { ni español }\end{array}$ & \\
\hline De acuerdo . . & 20,6 & 17,6 & 19,9 & 20,6 & 30,0 & 17,3 & 23,5 \\
\hline 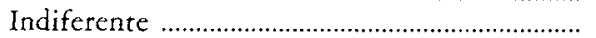 & 7,9 & 18,1 & 12,9 & 17,3 & 10,1 & 34,6 & 12,7 \\
\hline 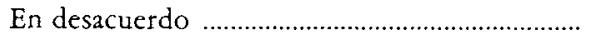 & 3,8 & 8,9 & 6,7 & 7,9 & 6,6 & 4,2 & 6,5 \\
\hline No la conoce & 67,7 & 55,3 & 60,5 & 54,3 & 49,4 & 40,0 & 57,2 \\
\hline TOTAL & 100 & 100 & 100 & 100 & 100 & 100 & \\
\hline
\end{tabular}

Fuente: Encuesta de GABISE, S. A., a la población de Cataluña, marzo 1988. 
Opinión sobre la provincia única en relación a la posición frente a la LOT

\begin{tabular}{|c|c|c|c|c|c|}
\hline \multirow{2}{*}{$\begin{array}{c}\text { Opinión sobre } \\
\text { la pronvicia unica }\end{array}$} & \multicolumn{4}{|c|}{ Opinión sobre la $L O T$} & \multirow[b]{2}{*}{ TOTAL } \\
\hline & De acuerdo & Indiferente & En desacuerdo & No la conoce & \\
\hline Favorable & 69,1 & 19,6 & 20,8 & 25,0 & 35,0 \\
\hline Indiferente & 9,8 & 62,6 & 11,2 & 50,8 & 39,4 \\
\hline Desfavorable.. & 21,1 & 17,8 & 68,6 & 24,2 & 25,6 \\
\hline TOTAL ............................ & 100 & 100 & 100 & 100 & 100 \\
\hline
\end{tabular}

FUENTE: Encuesta de GABISE, S. A., a la población de Cataluña, marzo 1988.

\section{CUADRO 9.II}

Opinión sobre la provincia única, según edad

\begin{tabular}{|c|c|c|c|c|c|c|c|}
\hline \multirow[b]{2}{*}{ Opinión sobre la provincia única } & \multicolumn{6}{|c|}{$E D A D$} & \multirow[b]{2}{*}{ TOTAL } \\
\hline & De 18 a 25 & De 26 a 35 & De 36 a 45 & De 46 a 55 & De $56 a 65$ & Más de 65 & \\
\hline 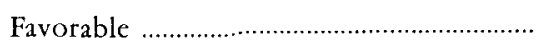 & 39,5 & 36,6 & 35,7 & 34,4 & 36,0 & 28,7 & 35,0 \\
\hline Indiferente & 35,5 & 34,1 & 35,0 & 38,7 & 42,4 & 51,3 & 39,6 \\
\hline 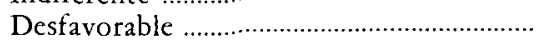 & 25,0 & 29,2 & 29,3 & 26,9 & 21,5 & 20,0 & 25,4 \\
\hline 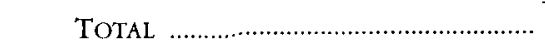 & 100 & 100 & 100 & 100 & 100 & 100 & 100 \\
\hline
\end{tabular}

Fuente: Encuesta de GABISE, S. A., a la población de Cataluña, marzo 1988. 


\section{CUADRO 10.II}

Opinión sobre la provincia única, según lugar de nacimiento

\begin{tabular}{|c|c|c|c|c|c|c|}
\hline \multirow[b]{2}{*}{ Opinión sobre la provincia única } & \multicolumn{5}{|c|}{ LUGAR DE NACIMIENTO } & \multirow[b]{2}{*}{ TOTAL } \\
\hline & Municipio & Provincia & $\begin{array}{c}\text { Resto de } \\
\text { Cataluña }\end{array}$ & $\begin{array}{l}\text { Resto de } \\
\text { España }\end{array}$ & Extranjero & \\
\hline Favorable & 39,7 & 38,7 & 37,4 & 27,4 & 25,0 & 34,9 \\
\hline 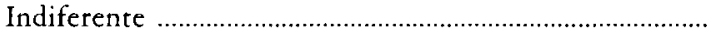 & 38,7 & 32,6 & 35,6 & 45,0 & 54,2 & 39,6 \\
\hline 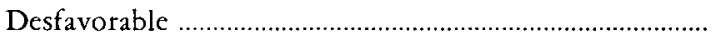 & 21,5 & 29,2 & 27,0 & 27,6 & 20,8 & 25,5 \\
\hline TOTAL …...................... & 100 & 100 & 100 & 100 & 100 & 100 \\
\hline
\end{tabular}

Fuente: Encuesta de GABISE, S. A., a la población de Cataluña, marzo 1988.

\section{CUADRO 11.II}

Opinión sobre la provincia única, según tamaño del municipio

\begin{tabular}{|c|c|c|c|c|c|c|}
\hline \multirow[b]{2}{*}{$\begin{array}{c}\text { Opinión sobre } \\
\text { la provincia única }\end{array}$} & \multicolumn{5}{|c|}{ NUMERO DE HABITANTES } & \multirow[b]{2}{*}{ TOTAL } \\
\hline & $\begin{array}{c}\text { Hasta } \\
2.000\end{array}$ & $\begin{array}{l}D e 2.001 \\
a 10.000\end{array}$ & $\begin{array}{c}\text { De } 10.001 \\
\text { a } 50.000\end{array}$ & $\begin{array}{l}\text { De } 50.001 \\
\text { a } 100.000\end{array}$ & $\begin{array}{l}\text { Más de } \\
100.000\end{array}$ & \\
\hline Favorable. & 42,5 & 38,6 & 40,3 & 51,2 & 28,9 & 35,0 \\
\hline Indiferente & 28,2 & 34,0 & 39,8 & 31,0 & 43,9 & 39,6 \\
\hline 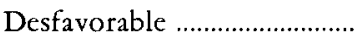 & 29,3 & 27,4 & 19,9 & 17,8 & 27,2 & 25,5 \\
\hline . & 100 & 100 & 100 & 100 & 100 & 100 \\
\hline
\end{tabular}

Fuente: Encuesta de GABISE, S. A., a la población de Cataluña, marzo 1988. 
Opinión sobre la provincia única, según conocimiento de la lengua que deberian tener los inmigrantes

\begin{tabular}{|c|c|c|c|c|c|}
\hline \multirow[b]{2}{*}{ Opinión sobre la provincia única } & \multicolumn{4}{|c|}{ CONOCIMIENTO DE LA LENGUA INMIGRANTES } & \multirow[b]{2}{*}{ TOTAL } \\
\hline & $\begin{array}{l}\text { Leer y } \\
\text { escribir }\end{array}$ & Hablar & Entender & $\begin{array}{l}\text { Ni bablar, } \\
\text { ni entender }\end{array}$ & \\
\hline Favorable ........ & 40,2 & 45,9 & 29,0 & 9,9 & 35,0 \\
\hline Indiferente & 29,9 & 29,3 & 48,2 & 52,2 & 29,6 \\
\hline 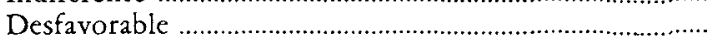 & 29,8 & 24,8 & 22,8 & 37,8 & 25,4 \\
\hline .......................... & 100 & 100 & 100 & 100 & 100 \\
\hline
\end{tabular}

FUENTE: Encuesta de GABISE, S. A., a la población de Cataluña, marzo 1988.

\section{CUADRO $13 . \mathrm{II}$}

Opinión sobre la provincia única, segün preferencia en las publicaciones de la Generalitat

\begin{tabular}{|c|c|c|c|c|}
\hline \multirow[b]{2}{*}{ Opinión sobre la provincia única } & \multicolumn{3}{|c|}{ Lengua preferida en las publicaciones } & \multirow[b]{2}{*}{ TOTAL } \\
\hline & Catalán & Castellano & Indiferente & \\
\hline 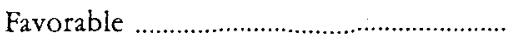 & 45,1 & 26,5 & 22,9 & 35,0 \\
\hline Indiferente & 32,6 & 42,7 & 51,3 & 39,5 \\
\hline 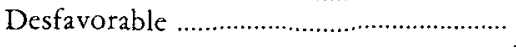 & 22,3 & 30,8 & 25,8 & 25,5 \\
\hline TOTAL & 100 & 100 & 100 & 100 \\
\hline
\end{tabular}

Fuente: Encuesta de GABISE, S. A. a la población de Cataluña, marzo 1988. 


\section{CUADRO 14.II}

Opinión sobre la provincia única, según el sentimiento predominante de identidad

\begin{tabular}{|c|c|c|c|c|c|c|c|}
\hline \multirow[b]{2}{*}{ Opinión sobre la provincia única } & \multicolumn{6}{|c|}{ SENTIMIENTO DE IDENTIDAD } & \multirow[b]{2}{*}{ TOTAL } \\
\hline & Español & $\begin{array}{l}\text { Más español } \\
\text { que catalán }\end{array}$ & $\begin{array}{c}\text { Tan español } \\
\text { como catalán }\end{array}$ & $\begin{array}{l}\text { Más catalán } \\
\text { que español }\end{array}$ & Catalàn & $\begin{array}{l}\text { Ni catalán, } \\
\text { ni español }\end{array}$ & \\
\hline (1) & 19,6 & 21,9 & 30,6 & 40,0 & 52,2 & 26,7 & 35,1 \\
\hline Indiferente & 50,5 & 39,4 & 42,6 & 36,7 & 30,1 & 41,7 & 39,6 \\
\hline 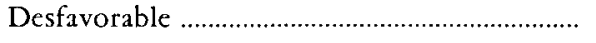 & 29,9 & 38,7 & 26,8 & 23,3 & 17,6 & 31,7 & 25,4 \\
\hline TOTAL & 100 & 100 & 100 & 100 & 100 & 100 & 100 \\
\hline
\end{tabular}

FUENTE: Encuesta de GABISE, S.A., a la población de Cataluña, marzo 1988. 


\section{A N E X O}

\section{CARACTERISTICAS TECNICAS DE LAS ENCUESTAS DE GABISE, S. A., DE LAS QUE PROVIENEN LOS DATOS MANEJADOS EN ESTA NOTA DE INVESTIGACION}

\section{A) Encuesta propia a la población de Barcelona, 1987}

Encuesta realizada por GABISE, S. A., mediante entrevista personal - en el domicilio del entrevistado- con cuestionario precodificado (con 31 variables categorizadas) durante los días 29 al 31 de mayo de 1987.

Trabajo de campo: Se ha revisado el 50 por 100 de las entrevistas y se han vuelto a realizar (comprobación) el 10 por 100 del total de casos.

Tratamiento estadístico: Ha sido realizado a partir del paquete estadístico BMDP, tanto para el análisis descriptivo como para las tablas de contingencia. Para la reasignación de indecisos, se ha utilizado el análisis discriminante.

Diseño de la muestra: Seleccionado aleatoriamente en base al Censo electoral actualizado en 1987. Estratificada por zonas de residencia. Con la previsión de un 50 por 100 de ampliación de la muestra como reservas para sustitución.

Universo: Ciudadanos de Barcelona, de ambos sexos, de dieciocho o más años.

Muestra real: 1.499 entrevistas, habiéndose realizado un 25 por 100 de sustituciones (despuès de tres intentos de localización).

Error muestral: Para una probabilidad (grado de confianza) del 95 por 100, el error muestral máximo es de 3,5 por 100.

\section{B) Encuesta propia a la población de Cataluña, febrero 1988}

Encuesta realizada por GABISE, S. A., mediante entrevista personal - en el domicilio del entrevistado- con cuestionario precodificado (22 preguntas y 29 variables) durante los días 8 al 13 de febrero.

Trabajo de campo: Comprobación de un 10 por 100 de las entrevistas.

Tratamiento estadístico: Realizado a partir del paquete BMDP, tanto para el análisis descriptivo como para las tablas de contingencia.

Diseño de la muestra: Seleccionada aleatoriamente por rutas, en un conjunto de municipios estratificados según tamaño y voto real en las elecciones autonómicas en Cataluña (1984); para las tipologías de los estratos se ha utilizado el análisis CLUSTER. Se han considerado cuatro subpoblaciones para obtener datos relativos a las cuatro circunscripciones electorales (provincias); también se ha realizado un estudio sociopolítico considerando una única población para el conjunto de Cataluña. 
Muestra real: El total de entrevistas realizadas ha sido de 2.425 .

Error muestral: Para una probabilidad (nivel de confianza) de 95 por 100 $(\mathrm{p}=\mathrm{q}=0,5)$, el error muestral máximo es de más/menos 3 por 100 para Barcelona $(\mathrm{n}=1.000)$; de más/menos 4 por 100 para Gerona, Lérida y Tarragona $(n=500)$, y de más/menos 2,5 por 100 para el conjunto de Cataluña $(n=1.490)$.

\section{C) Encuesta propia a la población de Cataluña, marzo 1988}

Encuesta realizada por GABISE, S. A., mediante entrevista personal -en el domicilio del entrevistado- con cuestionario precodificado (25 preguntas y 31 variables) durante los días 7 al 12 de marzo.

Trabajo de campo: Comprobación de un 10 por 100 de las entrevistas.

Tratamiento estadístico: Realizado a partir del paquete BMDP, tanto para el análisis descriptivo como para las tablas de contingencia.

Diseño de la muestra: Seleccionada aleatoriamente por rutas, sobre un conjunto de municipios estratificados según tamaño y voto real en las últimas elecciones autonómicas en Cataluña (1984); para las tipologías de los estratos se ha utilizado el análisis CLUSTER. Se han considerado cuatro subpoblaciones para obterner datos relativos a las cuatro circunscripciones electorales (provincias); también se ha realizado un estudio sociopolítico considerando una única población para el conjunto de Cataluña.

Muestra real: El total de entrevistas realizadas ha sido de 2.500 .

Error muestral: Para una probabilidad (nivel de confianza) del 95 por 100 $(p=q=0,5)$, el error muestral máximo es de más/menos 3 por 100 para Barcelona $(n=1.000)$; de más/menos 4 por 100 para Gerona, Lérida y Tarragona $(\mathrm{n}=500)$, y de más/menos 2 por 100 para el conjunto de Cataluña $(\mathrm{n}=2.500$, con una ponderación del peso respectivo de cada provincia). 
